\title{
Few-second-long correlation times in a quantum dot nuclear spin bath probed by frequency-comb nuclear magnetic resonance spectroscopy
}

\author{
A. M. Waeber ${ }^{1 \star}$, M. Hopkinson ${ }^{2}$, I. Farrer ${ }^{3}$, D. A. Ritchie ${ }^{3}$, J. Nilsson ${ }^{3}$, R. M. Stevenson ${ }^{4}$, A. J. Bennett ${ }^{4}$, \\ A. J. Shields ${ }^{4}$, G. Burkard ${ }^{5}$, A. I. Tartakovskii ${ }^{1}$, M. S. Skolnick ${ }^{1}$ and E. A. Chekhovich ${ }^{1 \star}$
}

One of the key challenges in spectroscopy is the inhomogeneous broadening that masks the homogeneous spectral lineshape and the underlying coherent dynamics. Techniques such as four-wave mixing and spectral hole-burning are used in optical spectroscopy ${ }^{1-3}$, and spin-echo ${ }^{4}$ in nuclear magnetic resonance (NMR). However, the high-power pulses used in spin-echo and other sequences ${ }^{4-8}$ often create spurious dynamics ${ }^{7,8}$ obscuring the subtle spin correlations important for quantum technologies $5^{5,6-17}$. Here we develop NMR techniques to probe the correlation times of the fluctuations in a nuclear spin bath of individual quantum dots, using frequency-comb excitation, allowing for the homogeneous NMR lineshapes to be measured without high-power pulses. We find nuclear spin correlation times exceeding one second in self-assembled InGaAs quantum dots-four orders of magnitude longer than in strain-free III-V semiconductors. This observed freezing of the nuclear spin fluctuations suggests ways of designing quantum dot spin qubits with a well-understood, highly stable nuclear spin bath.

Pulsed magnetic resonance is a diverse toolkit with applications in chemistry, biology and physics. In quantum information applications, solid state spin qubits are of great interest and are often described by the so-called central spin model, where the qubit (central spin) is coupled to a fluctuating spin bath (typically interacting nuclear spins). Here microwave and radiofrequency (rf) magnetic resonance pulses are used for the initialization and readout of a qubit ${ }^{18}$, dynamical decoupling ${ }^{5}$ and dynamic control ${ }^{6}$ of the spin bath.

However, the most important parameter controlling the central spin coherence $e^{9,11,19}$ - the correlation time $\tau_{c}$ of the spin bath fluctuations-is very difficult to measure directly. The value of $\tau_{c}$ is determined by the spin exchange (flip-flops) of the interacting nuclear bath spins. By contrast, pulsed NMR reveals the spin bath coherence time $T_{2}$, which characterizes the dynamics of the transverse nuclear magnetization ${ }^{7,8,20}$ and is much shorter than $\tau_{\mathrm{c}}$. The problem is further exacerbated in self-assembled quantum dots, where quadrupolar effects lead to inhomogeneous NMR broadening exceeding $10 \mathrm{MHz}$ (refs 21,22), so that pulsed NMR requires practically unattainable rf field amplitudes exceeding $1 \mathrm{~T}$.

Here we develop an alternative approach to NMR spectroscopy: we measure non-coherent depolarization of nuclear spins under weak noise-like rf fields. Contrary to intuitive expectation, we show that such measurement can reveal the full homogeneous NMR lineshape describing the coherent spin dynamics. This is achieved by employing rf excitation with a frequency-comb profile (widely used in precision optical metrology $y^{23}$ ). We then exploit non-resonant nuclear-nuclear interactions: the homogeneous NMR lineshape of one isotope measured with frequency-comb NMR is used as a sensitive non-invasive probe of the correlation times $\tau_{c}$ of the nuclear flip-flops of the other isotope. Although initial studies ${ }^{9,17,19}$ suggested $\tau_{\mathrm{c}} \sim 100 \mu$ s for nuclear spins in III-V semiconductors, it was recently recognized ${ }^{20,24,25}$ that quadrupolar effects may have a significant impact in self-assembled quantum dots. Here, for the first time, we obtain a quantitative measurement of extremely long $\tau_{\mathrm{c}} \gtrsim 1 \mathrm{~s}$, revealing strong freezing of the nuclear spin bath due to the inhomogeneous strain - a crucial advantage for quantum information applications of self-assembled quantum dots.

The experiments were performed on individual neutral selfassembled InGaAs/GaAs quantum dots at magnetic field $B_{z}=8 \mathrm{~T}$. All measurements of the nuclear spin depolarization dynamics employ the pump-depolarize-probe protocol shown in Fig. 1a. Here we exploit the hyperfine interaction of the nuclei with the optically excited electron ${ }^{17,21,22}$ both to polarize the nuclei (pump pulse) and to measure the nuclear spin polarization in terms of the Overhauser shift $\Delta E_{\mathrm{hf}}$ in the quantum dot photoluminescence spectrum (probe pulse). The rf magnetic field depolarizing nuclear spins is induced by a small copper coil. (Further experimental details can be found in Methods and Supplementary Note 1.)

All isotopes in the studied dots possess non-zero quadrupolar moments. Here we focus on the spin $I=3 / 2$ nuclei ${ }^{71} \mathrm{Ga}$ and ${ }^{75}$ As. The strain-induced quadrupolar shifts result in an inhomogeneously broadened NMR spectrum ${ }^{21,22}$, as shown schematically by the green line in Fig. 1b. The spectrum consists of a central transition (CT) $-1 / 2 \leftrightarrow+1 / 2$ and two satellite transition (ST) $\pm 1 / 2 \leftrightarrow \pm 3 / 2$ peaks. All nuclei in the dot are coupled by dipole-dipole interactions: an exact description of such a system is a very complex many-body quantum mechanical problem. Here we use a simplified semiclassical description where nuclear-nuclear interactions are taken into account by introducing a phenomenological homogeneous broadening of each nuclear spin transition. Thus, the NMR spectrum in Fig. $1 \mathrm{~b}$ is an inhomogeneous distribution (with width $\Delta v_{\text {inh }}$ ) of individual

${ }^{1}$ Department of Physics and Astronomy, University of Sheffield, Sheffield S3 7RH, UK. ${ }^{2}$ Department of Electronic and Electrical Engineering, University of Sheffield, Sheffield S1 3JD, UK. ${ }^{3}$ Cavendish Laboratory, University of Cambridge, Cambridge CB3 OHE, UK. ${ }^{4}$ Toshiba Research Europe Limited, Cambridge Research Laboratory, Cambridge CB4 OGZ, UK. ${ }^{5}$ Department of Physics, University of Konstanz, D-78457 Konstanz, Germany.

*e-mail: a.waeber@sheffield.ac.uk; e.chekhovich@sheffield.ac.uk 
a

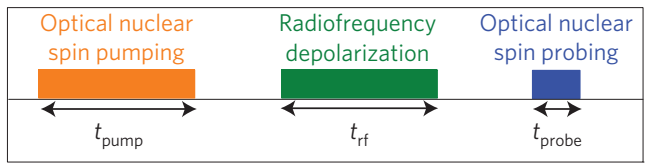

Experiment cycle

b

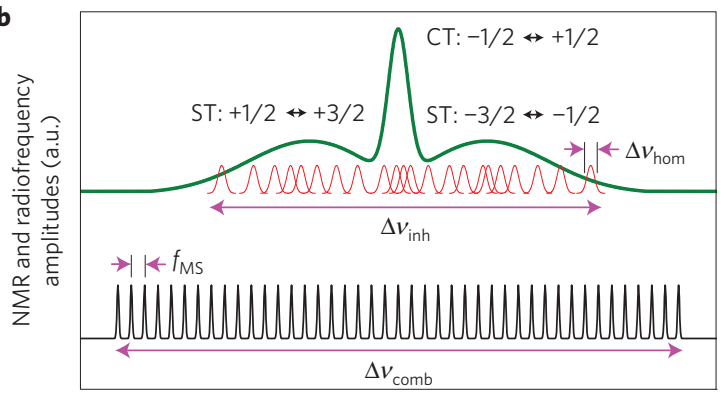

Radiofrequency, $v$

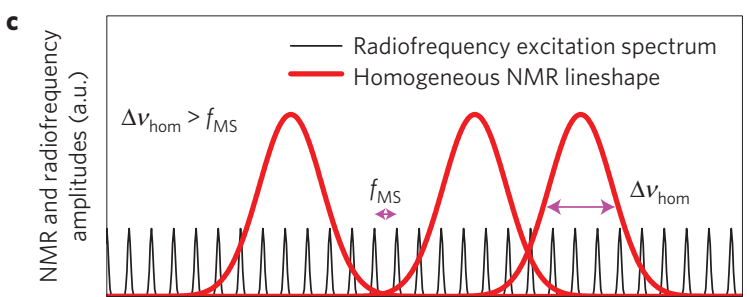

d

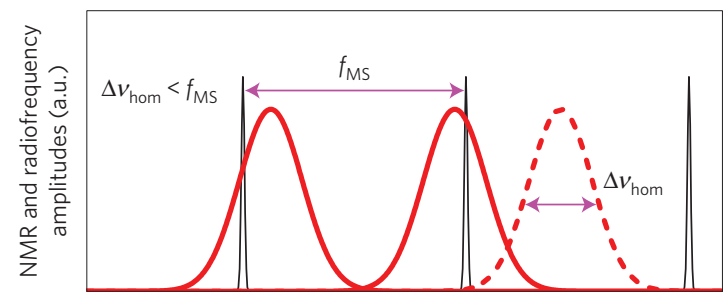

Radiofrequency, $v$

Figure 1 | Frequency-comb technique for homogeneous NMR lineshape measurement. a, Timing diagram of the experimental cycle consisting of nuclear spin optical pumping (duration $t_{\text {pump }}$ ), frequency-comb rf excitation $\left(t_{\mathrm{rf}}\right)$, and optical probing of the nuclear spin state $\left(t_{\text {probe }}\right)$. $\mathbf{b}$, The green line shows schematically a quantum dot NMR spectrum consisting of a central transition (CT) peak and two satellite transition (ST) bands. The inhomogeneous lineshape (width $\Delta v_{\text {inh }}$ ) is the sum of a large number $\left(10^{4}-10^{5}\right.$ ) of nuclear spin transitions with homogeneous linewidths $\Delta v_{\text {hom }}$ (shown with red lines). The black line shows the spectrum of the frequency-comb excitation with mode spacing $f_{M S}$ and total width $\Delta v_{\text {comb }}$ exceeding $\Delta v_{\text {inh }}$. c,d, Demonstrate how experiments with varying $f_{M S}$ can reveal the width $\Delta \nu_{\text {hom }}$ of the NMR homogeneous lineshape (red lines). When $\Delta v_{\text {hom }}>f_{M S}$ (c), all individual nuclei are uniformly excited by the frequency comb (shown with black lines). In the opposite case $\Delta v_{\text {hom }}<f_{\text {MS }}$ (d), some of the nuclei (dashed line) are not excited, resulting in a slow-down of nuclear spin dynamics. The transition between the two cases takes place when $f_{\mathrm{MS}} \sim \Delta v_{\text {hom }}$, allowing $\Delta \nu_{\text {hom }}$ to be measured.

nuclear spin transitions (shown by the red lines) with much smaller homogeneous linewidth $\Delta v_{\text {hom }}$.

Radiofrequency excitation is considered to be weak when nuclear spin precession is slower than transverse relaxation, resulting in non-coherent depolarization (that is without Rabi oscillations) of the nuclei ${ }^{26}$. To make such depolarization sensitive to the homogeneous NMR lineshape, we use rf excitation with a frequency-comb spectral profile. As shown in Fig. 1b (black line), the frequency comb has a mode spacing of $f_{\mathrm{MS}}$ and a total comb width $\Delta v_{\text {comb }}$ exceeding $\Delta v_{\text {inh }}$. The key idea of the frequency-comb technique is described in Fig. 1c,d, where two possible cases are shown. If the spacing is small $\left(f_{\mathrm{MS}} \ll \Delta v_{\text {hom }}\right.$, Fig. 1c) all nuclear transitions are excited by a large number of rf modes. As a result, all nuclear spins are depolarized at the same rate and we expect an exponential decay of the total nuclear spin polarization. In the opposite case of large mode separation $\left(f_{\mathrm{MS}}>\Delta v_{\text {hom }}\right.$, Fig. $\left.1 \mathrm{~d}\right)$, some of the nuclear transitions are out of resonance and are not excited (for example, the one shown by the dashed red line). As a result we expect a slowed-down non-exponential nuclear depolarization. The experiments are performed at different $f_{\mathrm{MS}}$; the $f_{\mathrm{MS}}$ for which a slow-down in depolarization is observed gives a measure of the homogeneous linewidth $\Delta v_{\text {hom }}$.

Experimental demonstration of this technique is shown in Fig. 2a. The Overhauser shift variation $\Delta E_{\mathrm{hf}}$ of ${ }^{71} \mathrm{Ga}$ is shown as a function of the depolarizing rf pulse duration $t_{\mathrm{rf}}$ for different $f_{\mathrm{MS}}$. For small values of $f_{\mathrm{MS}}=80$ and $435 \mathrm{~Hz}$ an exponential depolarization is observed. However, when $f_{\mathrm{MS}}$ is increased the depolarization becomes non-exponential and slows down significantly. The detailed dependence $\Delta E_{\mathrm{hf}}\left(t_{\mathrm{rf}}, f_{\mathrm{MS}}\right)$ is shown as a colour-coded plot in Fig. 2b. The threshold value of $f_{\mathrm{MS}}$ (marked with a white arrow) above which the nuclear spin dynamics becomes sensitive to the discrete structure of the frequency comb provides an estimate of $\Delta v_{\text {hom }} \sim 450 \mathrm{~Hz}$. Such a small homogeneous linewidth is detected in NMR resonances with inhomogeneous broadening of $\Delta v_{\text {inh }} \sim 6 \mathrm{MHz}$ (ref. 21), demonstrating the resolution power of frequency-comb non-coherent spectroscopy.

The information revealed by frequency-comb spectroscopy is not limited to rough estimates. An accurate determination of the linewidth and the shape of the tails of the full homogeneous lineshape is achieved with spin dynamics modelling based on rate equations (see details in Methods and Supplementary Note 2). We use the following two-parameter phenomenological model for the homogeneous lineshape:

$$
L(v) \propto\left(1+4(\sqrt[k]{2}-1) \frac{v^{2}}{\Delta v_{\mathrm{hom}}^{2}}\right)^{-k}
$$

where $\Delta v_{\text {hom }}$ is the homogeneous full-width at half-maximum and $k$ is a roll-off parameter that controls the tails (the behaviour of $L(v)$ at large $v$ ). For $k=1$ the lineshape corresponds to Lorentzian, whereas for $k \rightarrow \infty$ it tends to Gaussian: in this way equation (1) seamlessly describes the two most common lineshapes. Using $\Delta v_{\text {hom }}$ and $k$ as parameters we calculate the model $\Delta E_{\mathrm{hf}}\left(t_{\mathrm{rf}}, f_{\mathrm{MS}}\right)$ dependence and fit it to the experimental $\Delta E_{\mathrm{hf}}\left(t_{\mathrm{rf}}, f_{\mathrm{MS}}\right)$ to find a phenomenological description of the homogeneous NMR lineshape in self-assembled quantum dots.

The solid line in Fig. 3a shows the best-fit lineshape $\left(\Delta v_{\text {hom }} \approx 221 \mathrm{~Hz}\right.$ and $k \approx 1.67$ ) for the measurement shown in Fig. 2a,b. The dashed and dashed-dotted lines in Fig. 3a show for comparison the Lorentzian $(k=1)$ and Gaussian $(k \rightarrow \infty)$ lineshapes with the same $\Delta v_{\text {hom }}$. The difference in the lineshape tails is seen clearly in Fig. 3b, where a logarithmic scale is used. The model $\Delta E_{\mathrm{hf}}\left(t_{\mathrm{rf}}, f_{\mathrm{MS}}\right)$ dependence calculated with the best-fit parameters is shown in Fig. $3 c$ and with lines in Fig. $2 a-$ there is excellent agreement with experiment. By contrast, modelling $\Delta E_{\mathrm{hf}}\left(t_{\mathrm{rf}}, f_{\mathrm{MS}}\right)$ with Lorentzian (Fig. 3d) and Gaussian (Fig. 3e) lineshapes shows a pronounced deviation from the experiment, demonstrating the excellent sensitivity of the frequency-comb spectroscopy to the tails of the homogeneous spectral lineshape.

We have also performed frequency-comb NMR spectroscopy on ${ }^{75}$ As nuclei (Fig. 4a). Despite their larger inhomogeneous broadening, $\Delta v_{\text {inh }} \sim 18 \mathrm{MHz}$, the model fitting reveals even smaller $\Delta v_{\text {hom }} \approx 117 \mathrm{~Hz}$ and $k \approx 1.78$. From the $\Delta v_{\text {hom }}$ derived here we can estimate the nuclear spin coherence times $T_{2} \approx 1 /\left(\pi \Delta \nu_{\text {hom }}\right) \sim 1.4$ and $2.7 \mathrm{~ms}$ for ${ }^{71} \mathrm{Ga}$ and ${ }^{75} \mathrm{As}$, respectively. These values are in good agreement with the corresponding spin-echo $T_{2} \approx 1.2$ and $4.3 \mathrm{~ms}$ 

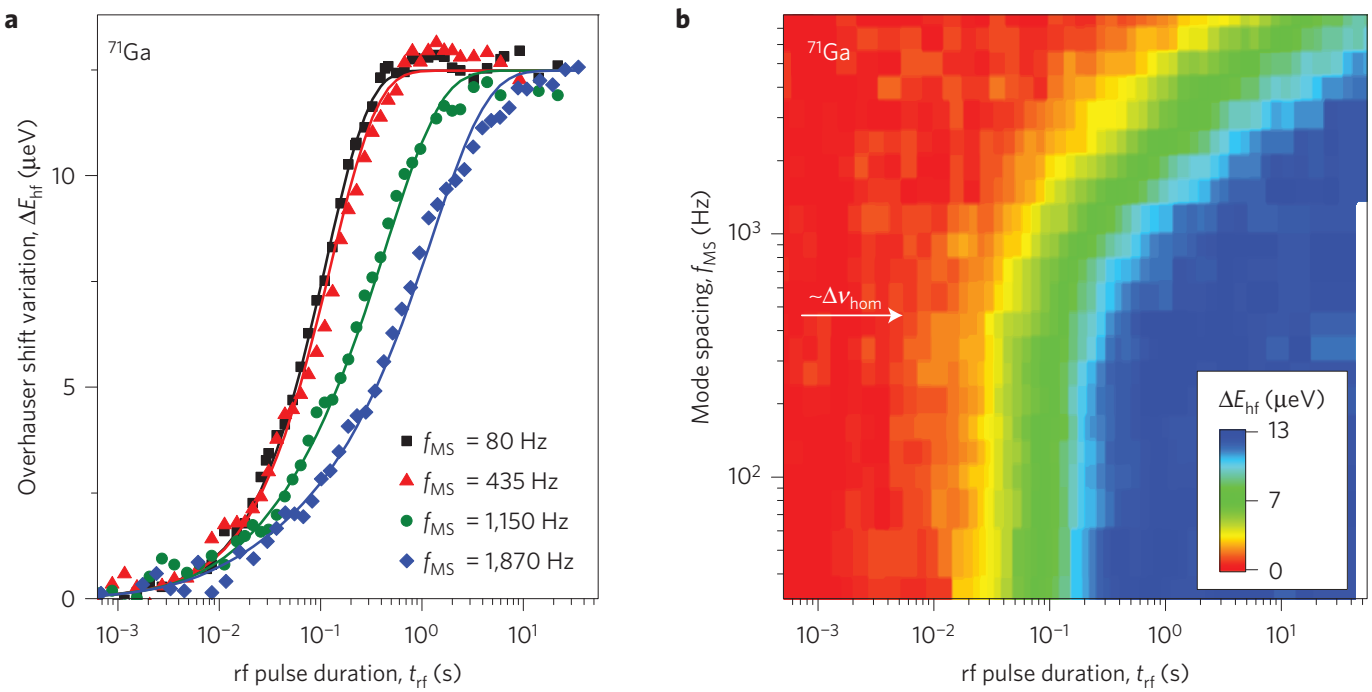

Figure 2 | Measurement of the homogeneous NMR lineshape in self-assembled quantum dots using frequency-comb excitation. a, The change in the polarization of the ${ }^{71} \mathrm{G}$ a nuclear spins (measured in terms of the change in the Overhauser shift $\Delta E_{\mathrm{hf}}$ ) is shown as a function of the rf pulse duration $t_{\mathrm{rf}}$ (symbols) at $B_{Z}=8 \mathrm{~T}$ and different mode spacings $f_{\mathrm{MS}}$. Lines show model fitting (see text). $\mathbf{b}, \mathrm{A}$ full $2 \mathrm{D}$ plot of $\Delta E_{\mathrm{hf}}$ as a function of $t_{\mathrm{rf}}$ and $f_{\mathrm{MS}}$ in the same experiment as in $\mathbf{a}$. There is a clear slow-down of the nuclear spin depolarization at $f_{M S}>450 \mathrm{~Hz}$ (shown with a white arrow), providing an estimate of the homogeneous linewidth of $\Delta v_{\text {hom }}$

derived in ref. 20. However, spin-echo could be measured only on the central transitions, for which $\Delta v_{\text {inh }}$ is relatively small. Moreover, pulsed NMR does not allow determination of the full homogeneous lineshape, which for dipole-dipole interactions typically has a 'top-hat'-like (Gaussian) profile ${ }^{27}$. And, most importantly, owing to parasitic effects such as 'instantaneous diffusion' and spin locking $^{8}$, pulsed NMR does not reveal the characteristic correlation time $\tau_{c}$ of the spin exchange flip-flops. In particular, owing to the 'instantaneous diffusion', the slow flip-flop dynamics become obscured by the much faster and trivial spin dynamics arising from a combined effect of secular spin-spin interactions and strong rf pulses ${ }^{7}$.

As we now show, the non-Gaussian lineshapes can be understood and $\tau_{c}$ can be derived using experiments with two frequency combs exciting nuclei of two isotopes $\left({ }^{75} \mathrm{As}\right.$ and $\left.{ }^{71} \mathrm{Ga}\right)$. We again excite ${ }^{75}$ As nuclei with a frequency comb to measure their homogeneous lineshape. The difference is that now we simultaneously apply a second comb exciting the ${ }^{71} \mathrm{Ga}$ spins. Importantly, in this experiment the ${ }^{71} \mathrm{Ga}$ nuclei are first fully depolarized after the optical nuclear spin pumping and before the two-comb excitation (see Supplementary Fig. 3) -in this way the excitation of ${ }^{71} \mathrm{Ga}$ has no direct effect on the measured hyperfine shift $\Delta E_{\mathrm{hf}}$. By contrast, it leads to 'heating' of the ${ }^{71} \mathrm{Ga}$ spins, which has only an indirect effect on $\Delta E_{\mathrm{hf}}$ by changing the ${ }^{75}$ As lineshape via dipolar coupling between ${ }^{71} \mathrm{Ga}$ and ${ }^{75} \mathrm{As}$ spins. The result of the two-comb experiment is shown in Fig. $4 \mathrm{~b}$ : a clear increase of $\Delta v_{\text {hom }}$ for ${ }^{75}$ As is observed. From model fitting we find that ${ }^{71} \mathrm{Ga}$ 'heating' leads to a three times broader homogeneous linewidth $\Delta v_{\text {hom }} \approx 355 \mathrm{~Hz}$ of ${ }^{75}$ As and its homogeneous lineshape is modified towards Gaussian, observed as an increase in $k \approx 2.32$.

To explain this result we note that the NMR lineshape is a statistical distribution of NMR frequency shifts of each nucleus produced by its dipolar interaction with all possible configurations of the neighbouring nuclear spins. However, in a frequency-comb experiment the evolution of the ${ }^{75} \mathrm{As}$ spin is limited in time by its depolarization. If the nuclear spin environment of each ${ }^{75} \mathrm{As}$ nucleus does not go through all possible configurations during the ${ }^{75}$ As characteristic depolarization time, the frequency shifts are effectively static, and hence are eliminated from the lineshape, as for any other inhomogeneous broadening.
Thus, we conclude that the narrowed, non-Gaussian $(k \approx 1.6-1.8)$ homogeneous NMR lineshape arises from the 'snapshot' nature of the frequency-comb measurement, probing the strongly frozen nuclear spin configuration. When the additional ${ }^{71} \mathrm{Ga}$ 'heating' excitation is applied it 'thaws' the ${ }^{71} \mathrm{Ga}$ spins, detected as broadening of the ${ }^{75}$ As lineshape (as demonstrated in Fig. 4a,b). If all isotopes were fully 'thawed' we would have observed a closeto-Gaussian $(k \gg 1)$ lineshape. By contrast, in the case of completely frozen flip-flops, the lineshape should be close to Lorentzian $(k=1)$, ultimately limited by the longitudinal nuclear spin relaxation. In a real experiment some spin fluctuations are always present: in particular the spins depolarized by the frequency comb influence one another via homonuclear dipolar interaction, resulting in a homogeneously broadened lineshape with some intermediate $k>1$. This interpretation is further supported by a microscopic Bloch equation model of the frequency-comb experiments, as discussed in Supplementary Note 5C.

We use the ${ }^{75}$ As lineshape as a probe to measure the dynamics of the ${ }^{71} \mathrm{Ga}$ equilibrium spin bath fluctuations. Rather than performing lengthy measurements of the entire ${ }^{75}$ As lineshape, we excite its spins with a frequency comb with a fixed $f_{\mathrm{MS}}=903 \mathrm{~Hz}$, for which the ${ }^{75} \mathrm{As}$ depolarization dynamics is most sensitive to the ${ }^{71} \mathrm{Ga}$ 'heating. We find that the resulting ${ }^{75} \mathrm{As}$ depolarization is well described by a stretched exponential function $\propto \exp \left[-\left(t_{\mathrm{rf}} / \tau_{\mathrm{As}}\right)^{r}\right]$ with $r \approx 0.6-0.9$ (see Methods); the measured value of $\tau_{\mathrm{As}}$ is used to characterize the response of ${ }^{75} \mathrm{As}$ to ${ }^{71} \mathrm{Ga}$ 'heating. Furthermore, we now use selective 'heating' of either the $-1 / 2 \leftrightarrow+1 / 2$ CT or the $+1 / 2 \leftrightarrow+3 / 2$ ST of ${ }^{71} \mathrm{Ga}$. The amplitude $\beta_{1, \mathrm{Ga}}$ of the 'heating' frequency comb is variedthe resulting dependences of the ${ }^{75} \mathrm{As}$ depolarization time $\tau_{\mathrm{As}}$ at different ${ }^{75}$ As comb amplitudes $\beta_{1, \mathrm{As}}$ are shown in Fig. $4 \mathrm{c}, \mathrm{d}$ for CT and ST 'heating', respectively. For analysis we also express $\beta_{1, \mathrm{Ga}}$ in terms of the rf-induced ${ }^{71} \mathrm{Ga}$ spin-flip time $\tau_{\mathrm{Ga}} \propto \beta_{1, \mathrm{Ga}}^{-2}$ (top scales in Fig. 4c,d, see details in Methods).

For vanishing ${ }^{71} \mathrm{Ga}$ excitation $\left(\beta_{1, \mathrm{Ga}}<2 \mathrm{nT} \mathrm{Hz}^{-1 / 2}, \tau_{\mathrm{Ga}}>20 \mathrm{~s}\right)$ the rf-induced spin-flip time $\tau_{\mathrm{Ga}}$ of ${ }^{71} \mathrm{Ga}$ is much larger than the correlation time $\tau_{\mathrm{c}}$ of the ${ }^{71} \mathrm{Ga}$ intrinsic spin flip-flops $\left(\tau_{\mathrm{Ga}}>\tau_{\mathrm{c}}\right)$. By contrast, strong 'heating' $\left(\beta_{1, \mathrm{Ga}}>200 \mathrm{nT} \mathrm{Hz}^{-1 / 2}\right.$, $\left.\tau_{\mathrm{Ga}}<2 \mathrm{~ms}\right)$ completely 'thaws' the ${ }^{71} \mathrm{Ga}$ spins $\left(\tau_{\mathrm{Ga}}<\tau_{\mathrm{c}}\right)$, broadens the ${ }^{75} \mathrm{As}$ lineshape (via heteronuclear interaction), and is observed as a reduction of $\tau_{\mathrm{As}}$. Another clear trend in Fig. $4 \mathrm{c}, \mathrm{d}$ is that the $\tau_{\mathrm{As}}\left(\beta_{1, \mathrm{Ga}}\right)$ 

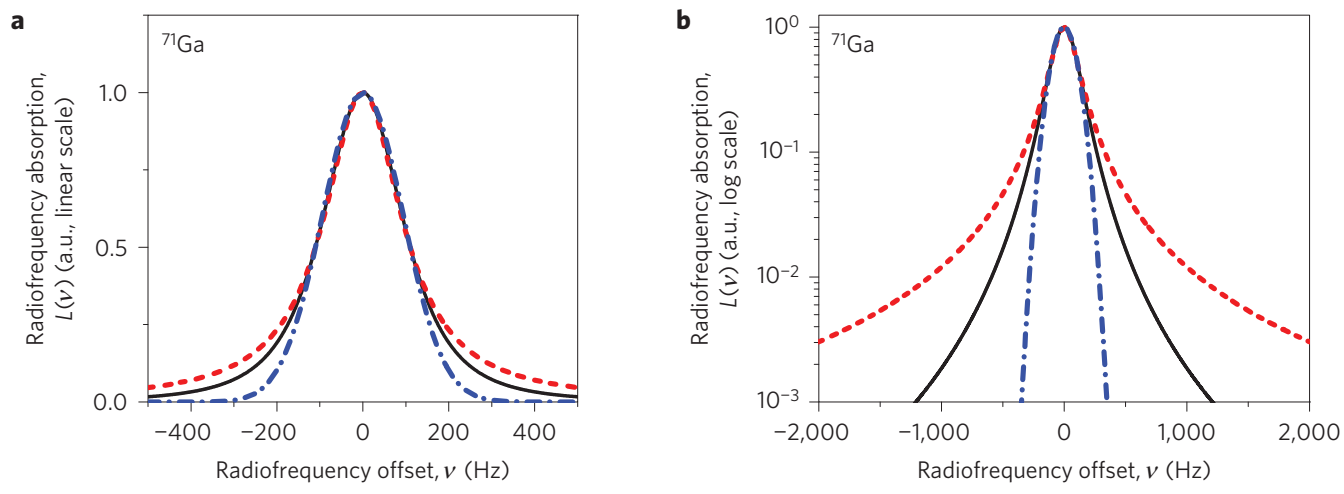

Fitted lineshape

-..- Lorentzian - -.-.- Gaussian
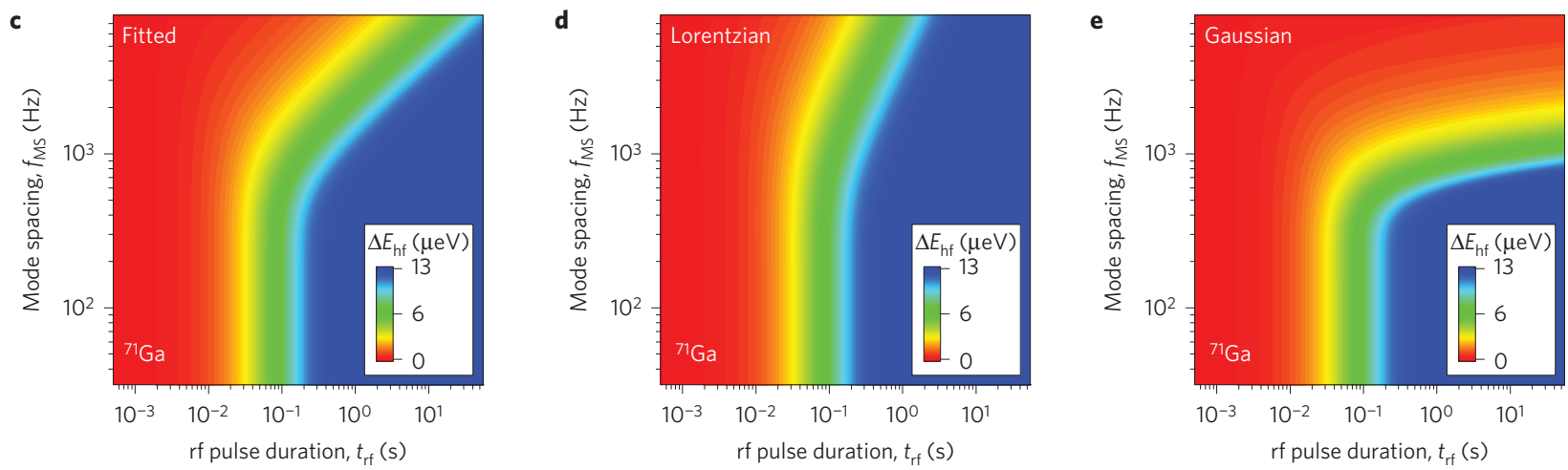

Figure 3 | Homogeneous lineshape modelling. a,b, Model homogeneous NMR lineshapes of ${ }^{71}$ Ga nuclei shown on linear (a) and logarithmic (b) scales. Solid lines show the best-fit lineshape with a full-width at half-maximum $\Delta v_{\text {hom }} \approx 221 \mathrm{~Hz}$ and a roll-off parameter $k \approx 1.67$. Dashed and dashed-dotted lines show for comparison Lorentzian and Gaussian lineshapes with the same $\Delta v_{\text {hom. }}$. c-e, The calculated $\Delta E_{\mathrm{hf}}\left(t_{\mathrm{rf}}, f_{\mathrm{MS}}\right)$ dependences for the lineshapes in a,b. For the fitted lineshape (c) an excellent agreement with the experiment in Fig. 2b is found, whereas calculations with Lorentzian (d) and Gaussian (e) lineshapes give markedly different results, demonstrating the sensitivity of the frequency-comb technique.

dependence becomes less pronounced at small $\beta_{1, \mathrm{As}}$. This is because ${ }^{75}$ As nuclei probe ${ }^{71} \mathrm{Ga}$ spin fluctuations on a timescale of the ${ }^{75} \mathrm{As}$ spin depolarization $\propto \beta_{1, \mathrm{As}}^{-2}$. At very small $\beta_{1, \mathrm{As}}$ the ${ }^{75}$ As spin evolution time becomes longer than $\tau_{\mathrm{c}}$, hence the ${ }^{75} \mathrm{As}$ lineshape is broadened by intrinsic ${ }^{71} \mathrm{Ga}$ fluctuations, and additional ${ }^{71} \mathrm{Ga}$ heating leads only to a minor reduction in $\tau_{\mathrm{As}}$. As we now show, this effect allows $\tau_{\mathrm{c}}$ to be deduced from the measurements with variable $\beta_{1, \mathrm{As}}$ and $\beta_{1, \mathrm{Ga}}$ shown in Fig. 4c,d.

First, as we show in Supplementary Note 5B, the heating-induced spin flips (with correlation time $\tau_{\mathrm{Ga}}$ ) and intrinsic dipolar flip-flops (with correlation time $\tau_{c}$ ) of ${ }^{71} \mathrm{Ga}$ have the same effect on ${ }^{75} \mathrm{As}$. Therefore, $\tau_{\mathrm{As}}$ depends only on the effective correlation time $\left(\tau_{\mathrm{Ga}}^{-1}+\tau_{\mathrm{c}}^{-1}\right)^{-1}$ of ${ }^{71} \mathrm{Ga}$ fluctuations. Second, as discussed above, ${ }^{75} \mathrm{As}$ homogeneous broadening is determined by fluctuations of ${ }^{71} \mathrm{Ga}$ only during the characteristic ${ }^{75} \mathrm{As}$ depolarization time. Consequently, the ${ }^{75}$ As homogeneous linewidth, and hence $\tau_{\mathrm{As}}$, depend only on the ratio of the ${ }^{71} \mathrm{Ga}$ effective correlation time and the ${ }^{75} \mathrm{As}$ characteristic evolution time $\left(\propto \beta_{1, \mathrm{As}}^{-2}\right)$. This ratio is proportional to $\beta_{1, \mathrm{As}}^{2}\left(\tau_{\mathrm{Ga}}^{-1}+\tau_{\mathrm{c}}^{-1}\right)^{-1}$ and $\tau_{\mathrm{As}}$ is its function. Although the analytical expression for this function is not known, it can be written as a Taylor series, from which we find that $\tau_{\mathrm{As}}$ depends linearly on $\left(\tau_{\mathrm{Ga}}^{-1}+\tau_{\mathrm{c}}^{-1}\right)^{-1}$ in the limit of small $\beta_{1, \mathrm{As}}$ (see details in Supplementary Note 4). Taking into account that $\left(\tau_{\mathrm{Ga}}^{-1}+\tau_{\mathrm{c}}^{-1}\right)^{-1} \in\left(0, \tau_{\mathrm{c}}\right)$ and $\left(\tau_{\mathrm{Ga}}^{-1}+\tau_{\mathrm{c}}^{-1}\right)^{-1}=\tau_{\mathrm{c}} / 2$ when $\tau_{\mathrm{c}}=\tau_{\mathrm{Ga}}$, we find that $\tau_{\mathrm{c}}$ can be estimated as the value of $\tau_{\mathrm{Ga}}$ for which $\tau_{\mathrm{As}}$ is a mean of its minimum and maximum values. From the measurements with the smallest $\beta_{1, \mathrm{As}}=3.4 \mathrm{nT} \mathrm{Hz}^{-1 / 2}$ we estimate in a straightforward way $\tau_{\mathrm{c}, \mathrm{CT}} \sim 0.5 \mathrm{~s}$ and $\tau_{\mathrm{c}, \mathrm{ST}} \sim 1.5 \mathrm{~s}$ for CT and ST respectively. A more accurate derivation of $\tau_{\mathrm{c}}$ is achieved by fitting the entire $\tau_{\mathrm{As}}$ data set with a stretched exponential function of $\beta_{1, \mathrm{As}}^{2}\left(\tau_{\mathrm{Ga}}^{-1}+\tau_{\mathrm{c}}^{-1}\right)^{-1}$, as shown by the lines in Fig. 4c,d (see details in Methods and Supplementary Note 4 ). Fitting yields correlation times $\tau_{c, \mathrm{CT}} \approx 1.0 \pm 0.2 \mathrm{~s}$ for CT and $\tau_{c, \mathrm{ST}} \approx 6.2 \pm 2.5 \mathrm{~s}$ for ST. Furthermore, in Supplementary Note 5 we reproduce the experimental data of Fig. $4 \mathrm{c}$ and $\mathrm{d}$ with a numerical model based on Bloch equations. We then successfully verify the fitting procedure used here to derive $\tau_{\mathrm{c}}$ by applying it to numerical model results with known $\tau_{c}$.

The observed $\tau_{c} \gtrsim 1 \mathrm{~s}$ greatly exceeds typical nuclear dipolar flipflop times in strain-free III-V solids $\tau_{c} \sim 100 \mu$ s (refs 9,10,17). We attribute the extremely long $\tau_{c}$ in self-assembled quantum dots to the effect of inhomogeneous nuclear quadrupolar shifts making nuclear spin flip-flops energetically forbidden ${ }^{20,24}$. This interpretation is corroborated by the observation of $\tau_{c, \mathrm{ST}}>\tau_{\mathrm{c}, \mathrm{CT}}$, because quadrupolar broadening of the ST transitions is much greater than that of the $\mathrm{CT}$ transitions (ref. 21). Furthermore, the ${ }^{71} \mathrm{Ga}$ spins examined here have the largest gyromagnetic ratio $\gamma$ and the smallest quadrupolar moment $Q$, so we expect that all other isotopes in InGaAs have even longer $\tau_{c}$, resulting in the overall $\tau_{c} \gtrsim 1 \mathrm{~s}$ of the entire quantum dot nuclear spin bath. This implies that in high magnetic fields the spin-echo coherence times of the electron and hole spin qubits in self-assembled dots are not limited by the nuclear spin bath up to sub-second regimes ${ }^{9,10,12,28,29}$. Therefore, the effort aimed at achieving III-V semiconductor quantum dot spin qubits with long coherence should be focused on understanding other mechanisms of central spin dephasing, such as electron-mediated nuclearnuclear interactions, nuclear spin dynamics induced by electron spin manipulation, or charge fluctuations ${ }^{13-15}$.

Because the frequency-comb technique is not limited by artefacts in the spin dynamics hampering pulsed magnetic resonance, it allows detection of very slow spin bath fluctuations. 

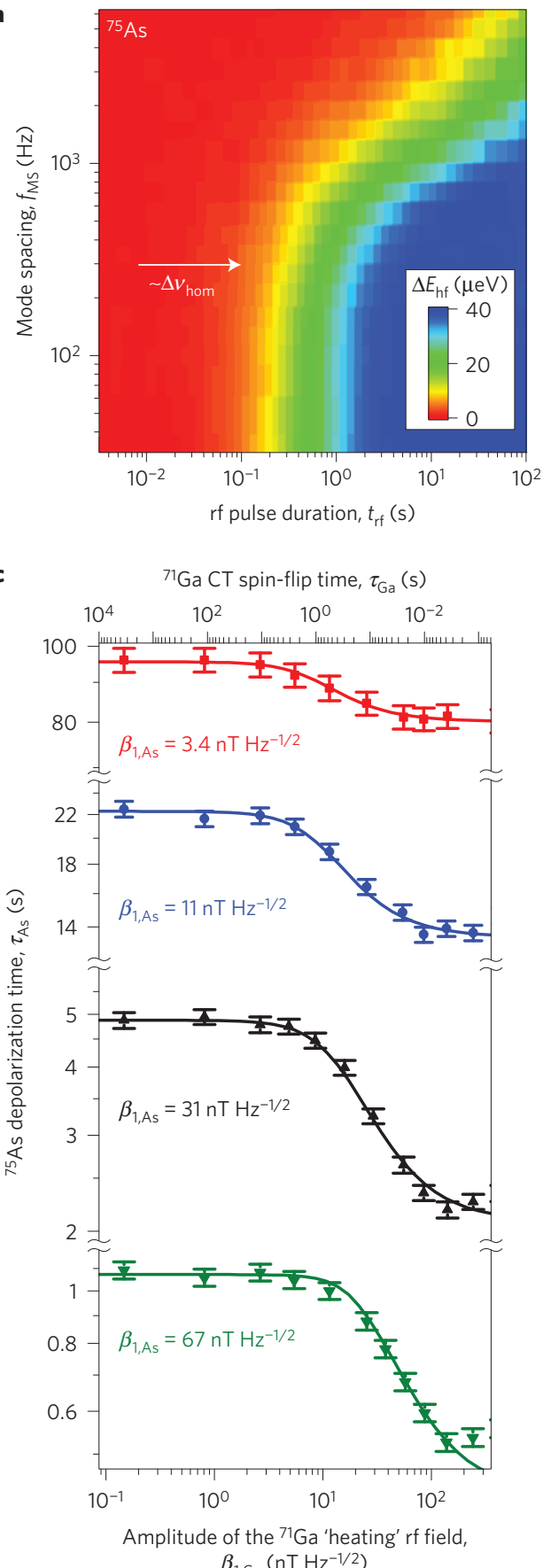

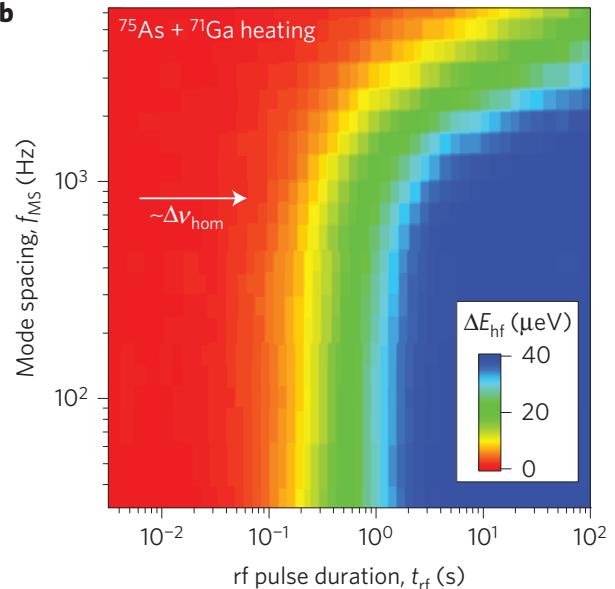

d d $\quad{ }^{71} \mathrm{GaST}$ spin-flip time, $\tau_{\mathrm{Ga}}(\mathrm{s})$

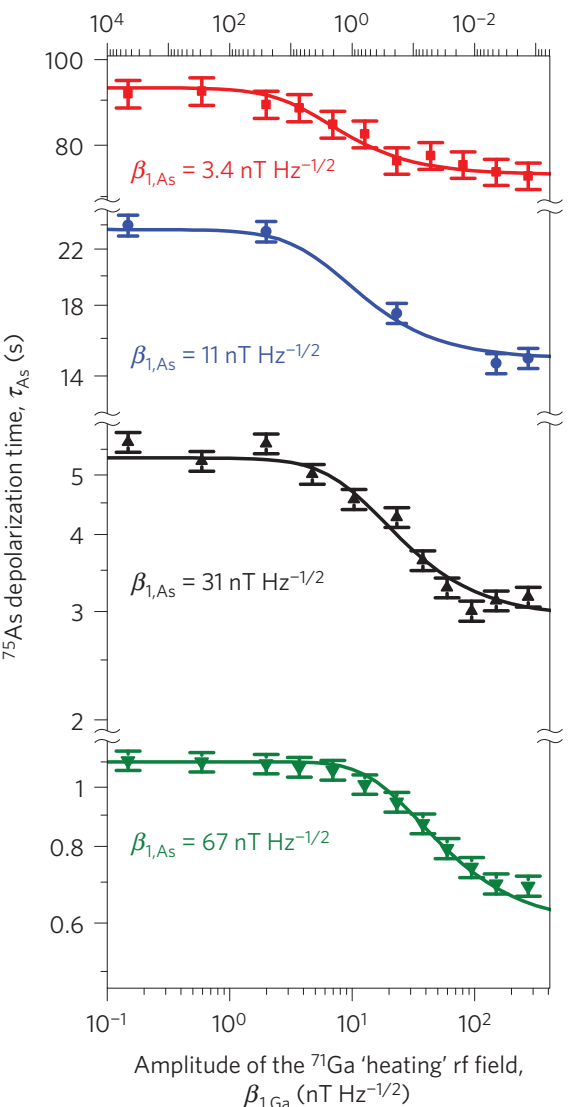

Figure 4 | Probing the correlation times of the nuclear spin bath fluctuation. $\mathbf{a}, \mathbf{b}$, Frequency-comb measurement on ${ }^{75}$ As nuclei at $B_{\mathrm{z}}=8 \mathrm{~T}$ without (a) and with (b) additional frequency-comb excitation 'heating' the ${ }^{71} \mathrm{G}$ a nuclei. The increase of $\Delta \nu_{\text {hom }}$ of ${ }^{75} \mathrm{As}$ under the ${ }^{71} \mathrm{Ga}$ 'heating' reveals the strongly suppressed nuclear spin fluctuations of ${ }^{71} \mathrm{Ga}$. c,d $\mathbf{d}$, Symbols show rf-induced depolarization time $\tau_{\mathrm{As}}$ of ${ }^{75} \mathrm{As}$ nuclei at fixed $f_{\mathrm{MS}}=903 \mathrm{~Hz}$ and four different amplitudes of the ${ }^{75} \mathrm{As}$ comb $\beta_{1, \mathrm{As}}$ as a function of the amplitude $\beta_{1, \mathrm{Ga}}$ of the additional rf excitation 'heating' either the central transition (CT, $\mathbf{c}$ ) or the satellite transition (ST, d) of ${ }^{71} \mathrm{Ga}$. Error bars show $95 \%$ confidence intervals. The top scales show $\beta_{1, \mathrm{Ga}}$ expressed in terms of the rf-induced spin-flip time $\tau_{\mathrm{Ga}}$ of ${ }^{71} \mathrm{Ga}$. The lines show model fitting from which we deduce the ${ }^{71} \mathrm{Ga}$ nuclear spin correlation times $\tau_{\mathrm{c}, \mathrm{CT}} \approx 1.0 \pm 0.2 \mathrm{~s}$ and $\tau_{\mathrm{c}, \mathrm{ST}} \approx 6.2 \pm 2.5 \mathrm{~s}$ for the $\mathrm{CT}$ and ST transitions.

Such sensitivity of the method can be used, for example, to investigate directly the effect of the electron or hole on the spin bath fluctuations in charged quantum dots-arising, for example, from hyperfine-mediated nuclear spin interactions. The experiments are well understood within a classical rate equation model and confirmed by a microscopic Bloch equation model, although further advances in frequency-comb spectroscopy can be expected with the development of a full quantum mechanical model.
Furthermore, the simple and powerful ideas of frequency-comb NMR spectroscopy can be readily extended beyond quantum dots: as we show in Supplementary Note 3, the only essential requirement is that the longitudinal relaxation time $T_{1}$ should be larger (by about two orders of magnitude) than the transverse relaxation time $T_{2}$, which is usually the case in solid state spin systems. Finally, our approaches in the use of frequency combs can go beyond NMR, enriching, for example, the techniques in optical spectroscopy. 


\section{Methods}

Methods and any associated references are available in the online version of the paper.

Received 14 June 2015; accepted 5 February 2016; published online 14 March 2016

\section{References}

1. Borri, P. et al. Ultralong dephasing time in InGaAs quantum dots. Phys. Rev. Lett. 87, 157401 (2001).

2. Volker, S. Hole-burning spectroscopy. Annu. Rev. Phys. Chem. 40, 499-530 (1989)

3. Yang, L. et al. Two-colour spin noise spectroscopy and fluctuation correlations reveal homogeneous linewidths within quantum-dot ensembles. Nature Commun. 5, 5949 (2014).

4. Hahn, E. L. Spin echoes. Phys. Rev. 80, 580-594 (1950).

5. Biercuk, M. J. et al. Optimized dynamical decoupling in a model quantum memory. Nature 458, 996-1000 (2009).

6. Bar-Gill, N. et al. Suppression of spin-bath dynamics for improved coherence of multi-spin-qubit systems. Nature Commun. 3, 858 (2012).

7. Tyryshkin, A. M. et al. Electron spin coherence exceeding seconds in high-purity silicon. Nature Mater. 11, 143-147 (2012).

8. Li, D., Dementyev, A. E., Dong, Y., Ramos, R. G. \& Barrett, S. E. Generating unexpected spin echoes in dipolar solids with $\pi$ pulses. Phys. Rev. Lett. 98, 190401 (2007).

9. Merkulov, I. A., Efros, A. L. \& Rosen, M. Electron spin relaxation by nuclei in semiconductor quantum dots. Phys. Rev. B 65, 205309 (2002).

10. de Sousa, R. \& Das Sarma, S. Theory of nuclear-induced spectral diffusion: spin decoherence of phosphorus donors in Si and GaAs quantum dots. Phys. Rev. B 68, 115322 (2003).

11. Yao, W., Liu, R.-B. \& Sham, L. J. Theory of electron spin decoherence by interacting nuclear spins in a quantum dot. Phys. Rev. B 74, 195301 (2006).

12. Bluhm, H. et al. Dephasing time of GaAs electron-spin qubits coupled to a nuclear bath exceeding $200 \mu$ s. Nature Phys. 7, 109-113 (2011).

13. Press, D. et al. Ultrafast optical spin echo in a single quantum dot. Nature Photon. 4, 367-370 (2010).

14. De Greve, K. et al. Ultrafast coherent control and suppressed nuclear feedback of a single quantum dot hole qubit. Nature Phys. 7, 872-878 (2011).

15. Greilich, A., Carter, S. G., Kim, D., Bracker, A. S. \& Gammon, D. Optical control of one and two hole spins in interacting quantum dots. Nature Photon. 5, 702-708 (2011).

16. Hansom, J. et al. Environment-assisted quantum control of a solid-state spin via coherent dark states. Nature Phys. 10, 1745-2473 (2014).

17. Urbaszek, B. et al. Nuclear spin physics in quantum dots: an optical investigation. Rev. Mod. Phys. 85, 79-133 (2013).

18. Cai, J., Retzker, A., Jelezko, F. \& Plenio, M. B. A large-scale quantum simulator on a diamond surface at room temperature. Nature Phys. 9, 168-173 (2013).
19. de Sousa, R. \& Das Sarma, S. Electron spin coherence in semiconductors: considerations for a spin-based solid-state quantum computer architecture. Phys. Rev. B 67, 033301 (2003).

20. Chekhovich, E. A., Hopkinson, M., Skolnick, M. S. \& Tartakovskii, A. I. Suppression of nuclear spin bath fluctuations in self-assembled quantum dots induced by inhomogeneous strain. Nature Commun. 6, 6348 (2015).

21. Chekhovich, E. A. et al. Structural analysis of strained quantum dots using nuclear magnetic resonance. Nature Nanotech. 7, 646-650 (2012).

22. Munsch, M. et al. Manipulation of the nuclear spin ensemble in a quantum dot with chirped magnetic resonance pulses. Nature Nanotech. 9, 671-675 (2014).

23. Udem, T., Holzwarth, R. \& Hansch, T. W. Optical frequency metrology. Nature 416, 233-237 (2002)

24. Dzhioev, R. I. \& Korenev, V. L. Stabilization of the electron-nuclear spin orientation in quantum dots by the nuclear quadrupole interaction. Phys. Rev. Lett. 99, 037401 (2007).

25. Latta, C., Srivastava, A. \& Imamoglu, A. Hyperfine interaction-dominated dynamics of nuclear spins in self-assembled InGaAs quantum dots. Phys. Rev. Lett. 107, 167401 (2011).

26. Madhu, P. \& Kumar, A. Direct cartesian-space solutions of generalized Bloch equations in the rotating frame. J. Magn. Reson. Ser. A 114, 201-211 (1995).

27. Van Vleck, J. H. The dipolar broadening of magnetic resonance lines in crystals. Phys. Rev. 74, 1168-1183 (1948).

28. Khaetskii, A. V., Loss, D. \& Glazman, L. Electron spin decoherence in quantum dots due to interaction with nuclei. Phys. Rev. Lett. 88, 186802 (2002).

29. Bechtold, A. et al. Three-stage decoherence dynamics of an electron spin qubit in an optically active quantum dot. Nature Phys 11, 1005-1008 (2015).

\section{Acknowledgements}

The authors are grateful to K. V. Kavokin for useful discussions. This work has been supported by the EPSRC Programme Grant EP/J007544/1, ITN S ${ }^{3}$ NANO. E.A.C. was supported by a University of Sheffield Vice-Chancellor's Fellowship and a Royal Society University Research Fellowship. I.F. and D.A.R. were supported by EPSRC.

Computational resources were provided in part by the University of Sheffield HPC cluster 'Iceberg.

\section{Author contributions}

M.H., I.F., D.A.R., J.N., R.M.S., A.J.B. and A.J.S. developed and grew the samples. A.M.W. and E.A.C. conceived and designed the experiments and analysed the data. A.M.W. performed the experiments. E.A.C. performed the numerical modelling. E.A.C., A.M.W., M.S.S., A.I.T., G.B. and A.J.B. wrote the manuscript with input from all authors. E.A.C. coordinated the project.

\section{Additional information}

Supplementary information is available in the online version of the paper. Reprints and permissions information is available online at www.nature.com/reprints.

Correspondence and requests for materials should be addressed to A.M.W. or E.A.C.

\section{Competing financial interests}

The authors declare no competing financial interests. 


\section{Methods}

Sample structures and experimental techniques. The experiments were performed on individual neutral self-assembled InGaAs/GaAs quantum dots. The sample was mounted in a helium-bath cryostat $(T=4.2 \mathrm{~K})$ with a magnetic field $B_{\mathrm{z}}=8 \mathrm{~T}$ applied in the Faraday configuration (along the sample growth and light propagation direction $\mathrm{Oz}$ ). A radiofrequency (rf) magnetic field $B_{\mathrm{rf}}$ perpendicular to $B_{z}$ was induced by a miniature copper coil. Optical excitation was used to induce nuclear spin magnetization exceeding $50 \%$, as well as to probe it by measuring hyperfine shifts in photoluminescence spectroscopy ${ }^{21}$.

Two sample structures have been studied, both containing a single layer of InGaAs/GaAs quantum dots embedded in a weak planar microcavity with a Q-factor of $\sim 250$. In one of the samples the dots emitting at $\sim 945 \mathrm{~nm}$ were placed in a $p-i-n$ structure, where application of a large reverse bias during the $\mathrm{rf}$ excitation ensured the neutral state of the dots. The results for this sample are shown in Fig. 2. The second sample was a gate-free structure, where most of the dots emitting at $\sim 914 \mathrm{~nm}$ are found in a neutral state, although the charging can not be controlled. Excellent agreement between the lineshapes of both ${ }^{71} \mathrm{Ga}$ and ${ }^{75} \mathrm{As}$ in the two structures was found, confirming the reproducibility of the frequency-comb technique. The results for this second sample are shown in Fig. 4.

Rate equation model for homogeneous lineshape derivation. Let us consider an ensemble of spin $I=1 / 2$ nuclei with gyromagnetic ratio $\gamma$ and inhomogeneously broadened distribution of nuclear resonant frequencies $v_{\text {nuc }}$. We assume that each nucleus has a homogeneous absorption lineshape $L(v)$, with normalization $\int_{-\infty}^{+\infty} L(v) d v=1$. A small amplitude (non-saturating) rf field will result in depolarization, which can be described by a differential equation for population probabilities $p_{ \pm 1 / 2}$ of the nuclear spin levels $I_{z}= \pm 1 / 2$

$$
\mathrm{d}\left(p_{+1 / 2}-p_{-1 / 2}\right) / \mathrm{d} t=-W\left(p_{+1 / 2}-p_{-1 / 2}\right)
$$

For frequency-comb excitation the decay rate is the sum of the decay rates caused by each $\mathrm{rf}$ mode with magnetic field amplitude $B_{1}$, and can be written as:

$$
W\left(v_{\text {nuc }}\right)=\frac{\gamma^{2} B_{1}^{2}}{2 f_{\mathrm{MS}}} \sum_{j=0}^{N_{\mathrm{m}}-1} L\left(v_{\mathrm{nuc}}-v_{1}-j f_{\mathrm{MS}}\right) f_{\mathrm{MS}}
$$

where the summation goes over all modes with frequencies $v_{\mathrm{j}}=v_{1}+j f_{\mathrm{MS}}\left(v_{1}\right.$ is the frequency of the first spectral mode).

The change in the Overhauser shift $E_{\mathrm{hf}}$ produced by each nucleus is proportional to $p_{+1 / 2}-p_{-1 / 2}$ and, according to equation (2) has an exponential time dependence $\propto \exp \left(-W\left(v_{\text {nuc }}\right) t\right)$. The quantum dot contains a large number of nuclear spins with randomly distributed absorption frequencies. Therefore, to obtain the dynamics of the total Overhauser shift we need to average over $v_{\text {nuc }}$. Because the spectrum of the rf excitation is periodic and $\Delta v_{\text {inh }} \gg f_{\mathrm{MS}}$, the averaging can be done over one period $f_{\text {MS }}$. Furthermore, because the total width of the rf frequency comb $\Delta v_{\text {comb }}$ is much larger than $f_{\mathrm{MS}}$ and $\Delta v_{\text {hom }}$, the summation in equation (3) can be extended to $\pm \infty$. Thus, the following expression is obtained for the time dependence $\Delta E_{\mathrm{hf}}\left(t, f_{\mathrm{MS}}\right)$, describing the dynamics of the rf-induced nuclear spin depolarization:

$$
\frac{\Delta E_{\mathrm{hf}}\left(t, f_{\mathrm{MS}}\right)}{\Delta E_{\mathrm{hf}}(t \rightarrow \infty)}=1-f_{\mathrm{MS}}^{-1} \int_{0}^{f_{\mathrm{MS}}} \exp \left(-t \frac{\gamma^{2} B_{1}^{2}}{2 f_{\mathrm{MS}}} \sum_{j=-\infty}^{\infty} L\left(v_{\mathrm{nuc}}-j f_{\mathrm{MS}}\right) f_{\mathrm{MS}}\right) d v_{\mathrm{nuc}}
$$

Equation (4) describes the dependence $\Delta E_{\mathrm{hf}}\left(t, f_{\mathrm{MS}}\right)$ directly measurable in experiments such as shown in Fig. 2b. $\Delta E_{\mathrm{hf}}(t \rightarrow \infty)$ is the total optically induced Overhauser shift of the studied isotope and is also measurable, whereas $f_{\mathrm{MS}}$ and $B$ are parameters that are controlled in the experiment. We note that in the limit of small mode spacing, $f_{\mathrm{MS}} \rightarrow 0$, the infinite sum in equation (4) tends to the integral $\int_{-\infty}^{+\infty} L(v) d v=1$ and the Overhauser shift decay is exponential (as observed experimentally) with a characteristic time

$$
\tau=2 f_{\mathrm{MS}} /\left(\gamma^{2} B_{1}^{2}\right)
$$

Equation (4) is a Fredholm integral equation of the first kind on the homogeneous lineshape function $L(v)$. This is an ill-conditioned problem: as a result finding the lineshape requires some constraints to be placed on $L(v)$. Our approach is to use the model lineshape of equation (1). After substituting $L(v)$ from equation (1), the right-hand side of equation (4) becomes a function of the parameters $\Delta \nu_{\text {hom }}$ and $k$, which we then find by least-squares fitting of equation (4) to the experimental dependence $\Delta E_{\mathrm{hf}}\left(t, f_{\mathrm{MS}}\right)$.

This model is readily extended to the case of $I>1 / 2$ nuclei. Equation (2) becomes a tri-diagonal system of differential equations, and the solution (equation (3)) contains a sum of multiple exponents under the integral. These modifications are straightforward but tedious, and can be found in Supplementary Note 2.

Derivation of the nuclear spin bath correlation times. Accurate lineshape modelling is crucial in revealing the ${ }^{75}$ As homogeneous broadening arising from ${ }^{71} \mathrm{Ga}$ 'heating' excitation (as demonstrated in Fig. 4a,b). However, because a measurement of the full $\Delta E_{\mathrm{hf}}\left(t, f_{\mathrm{MS}}\right)$ dependence is time consuming, the experiments with variable comb amplitudes $\beta_{1, \mathrm{Ga}}$ and $\beta_{1, \mathrm{As}}$ (Fig. $4 \mathrm{c}, \mathrm{d}$ ) were conducted at a fixed $f_{\mathrm{MS}}=903 \mathrm{~Hz}$ well in excess of the ${ }^{75}$ As homogeneous linewidth $\Delta v_{\text {hom }} \approx 117 \mathrm{~Hz}$. To extract the ${ }^{75}$ As depolarization time $\tau_{\text {As }}$ we fit the ${ }^{75} \mathrm{As}$ depolarization dynamics $\Delta E_{\mathrm{hf}}(t)$ with the following formula: $\Delta E_{\mathrm{hf}}\left(t_{\mathrm{rf}}\right)=\Delta E_{\mathrm{hf}}\left(t_{\mathrm{rf}} \rightarrow \infty\right) \times\left(1-\exp \left[-\left(t_{\mathrm{rf}} / \tau_{\mathrm{As}}\right)^{r}\right]\right)$, using $r$ as a common fitting parameter and $\tau_{\mathrm{As}}$ independent for measurements with different $\tau_{\mathrm{Ga}}$. We find $r \approx 0.6-0.9$, depending on $\beta_{1, \mathrm{As}}$, and the dependence of $\tau_{\mathrm{As}}$ on $\beta_{1, \mathrm{Ga}}$ obtained from the fit is shown in Fig. $4 \mathrm{c}, \mathrm{d}$ by the symbols with error bars corresponding to $95 \%$ confidence intervals.

The spacing of the ${ }^{71} \mathrm{Ga}$ 'heating' frequency comb is kept at a small value, $f_{\mathrm{MS}}=150 \mathrm{~Hz}$, ensuring uniform excitation of all nuclear spin transitions. The amplitude of the 'heating' comb is defined as $\beta_{1, \mathrm{Ga}}=B_{1} / \sqrt{f_{\mathrm{MS}}}$, where $B_{1}$ is magnetic field amplitude of each mode in the comb (further details can be found in Supplementary Note 1). To determine the correlation times we express $\beta_{1, \mathrm{Ga}}$ in terms of the rf-induced spin-flip time $\tau_{\mathrm{Ga}}$. The $\tau_{\mathrm{Ga}}$ is defined as the exponential time of the ${ }^{71} \mathrm{Ga}$ depolarization induced by the 'heating' comb, and is derived from an additional calibration measurement. The values of $\beta_{1, \mathrm{Ga}}$ shown in Fig. $4 \mathrm{c}$ are calculated using equation (5) as $\sqrt{2 /\left(4 \gamma_{\mathrm{Ga}}^{2} \tau_{\mathrm{Ga}}\right)}$, where $\gamma_{\mathrm{Ga}}$ is the ${ }^{71} \mathrm{Ga}$ gyromagnetic ratio and $\tau_{\mathrm{Ga}}$ is experimentally measured. The additional factor of 4 in the denominator is due to the matrix element of the CT of $\operatorname{spin} I=3 / 2$. For experiments on ST the $\beta_{1, \mathrm{Ga}}$ values shown in Fig. $4 \mathrm{~d}$ are obtained with the same formulae, but multiplied by $\sqrt{4 / 3}$.

The effect of ${ }^{71} \mathrm{Ga}$ heating on ${ }^{75} \mathrm{As}$ (probed with $\tau_{\mathrm{As}}$ ) is controlled by the ratio of the ${ }^{71} \mathrm{Ga}$ effective correlation time $\left(\tau_{\mathrm{Ga}}^{-1}+\tau_{\mathrm{c}}^{-1}\right)^{-1}$ and the characteristic time of ${ }^{75} \mathrm{As}$ rf-induced depolarization $2 /\left(\gamma_{\mathrm{As}}^{2} \beta_{1, \mathrm{As}}^{2}\right)$ given by equation (5). The experimental data of Fig. $4 \mathrm{c}, \mathrm{d}$ are fitted with the function $\tau_{\mathrm{As}}=\tau_{\mathrm{As}, \min }\left(\Delta \tau_{\mathrm{As}}+\left(1-\Delta \tau_{\mathrm{As}}\right)\right.$ $\left.\exp \left[-\left(\gamma_{\mathrm{As}}^{2} \beta_{1, \mathrm{As}}^{2}\left(\tau_{\mathrm{Ga}}^{-1}+\tau_{\mathrm{c}}^{-1}\right)^{-1} /(2 \theta)\right)^{s}\right]\right)$, where $\theta \approx 0.4, s \approx 0.6$ are dimensionless parameters describing the modified (stretched) exponential function, $\Delta \tau_{\mathrm{As}} \approx 2$ is the ratio of $\tau_{\mathrm{As}}$ without and with strong ${ }^{71} \mathrm{Ga}$ heating, $\tau_{\mathrm{As}, \min }$ is $\tau_{\mathrm{As}}$ in the limit of large $\beta_{1, \mathrm{Ga}}$, dependent on $\beta_{1, \mathrm{As}}$, and $\tau_{\mathrm{c}}$ is the correlation time of the ${ }^{71} \mathrm{Ga}$ spin fluctuations. Further details of the analysis used to derive $\tau_{c}$ from experimental data can be found in Supplementary Note 4. Moreover, we have verified this fitting procedure by applying it to numerically simulated spin dynamics with known $\tau_{\mathrm{c}}$, as described in Supplementary Note 5D. 